\title{
On Hilbert I-convergent sequence spaces
}

\author{
Vakeel A. Khan ${ }^{\mathrm{a}, *}$, Kamal M. A. S. Alshlool ${ }^{\mathrm{a}}$, Masood Alam ${ }^{\mathrm{b}}$ \\ a Department of Mathematics, Aligarh Muslim University, Aligarh, 202002, India. \\ ${ }^{b}$ Department of Mathematics and IT Center for Preparatory Studies, Sultan Qaboos University, P. O. Box 162-PC, 123 Al Khoud Muscat, \\ Sultanate of Oman.
}

\begin{abstract}
In this paper, we define some new sequence spaces $c_{0}^{I}(H), c^{I}(H), \ell_{\infty}^{I}(H)$, and $\ell_{\infty}(H)$ as a domain of triangle Hilbert matrix and study some topological and algebraic properties of these spaces. Further, we study some inclusion relations concerning these spaces.
\end{abstract}

Keywords: Hilbert matrix, Hilbert I-convergence, Hilbert I-Cauchy, Hilbert I-bounded.

2010 MSC: 40A35, 40C05, 47B37.

(C)2020 All rights reserved.

\section{Introduction}

Let $\mathbb{N}$ and $\mathbb{R}$, denote the sets of all natural and real numbers, respectively. By $\omega$, we denotes the vector space of all real or complex valued sequences. Any vector subspace of $\omega$ is called a sequence space. A family of sets $I \subset P(X)$ (where $P(X)$ is the power set of a non-empty set $X$ ) of subsets of $X$ is said to be ideal in $X$ if and only if (i) $\emptyset \in I$, (ii) for each $A, B \in I$ we have $A \cup B \in I$, (iii) for each $A \in I$ and $B \subset A$ we have $B \in I$ and $I$ is called an admissible in $X$ if and only if $I \neq X$ and it contains all singletons. A filter on $X$ is a non-empty family of sets $\mathcal{F} \subset P(X)$ satisfying (i) $\emptyset \notin \mathcal{F}$, (ii) for each $A, B \in \mathcal{F}$ we have $A \cap B \in \mathcal{F}$, (iii) for each $A \in \mathcal{F}$ and $B \supset A$ we have $B \in \mathcal{F}$. For each ideal $I$ there is a filter $\mathcal{F}(\mathrm{I})$ corresponding to $\mathrm{I}$, that is, $\mathcal{F}(\mathrm{I})=\left\{\mathrm{K} \subseteq X: \mathrm{K}^{\mathrm{c}} \in \mathrm{I}\right\}$, where $\mathrm{K}^{\mathrm{c}}=\mathrm{X} \backslash \mathrm{K}$. Depending on the structure of ideals of subsets of $\mathbb{N}$, Kostyrko et al. [18] defined the notion of I-convergence as a generalization of statistical convergence introduced by Fast [6] and Steinhaus [25]. Later, the notion of I-convergence was further investigated from the sequence space point of view and linked with the summability theory by Šalát et al. [23, 24], Khan and Nazneen [15], Khan et al. [17], Filipów and Tryba [7] and many other authors. For further details on ideal convergence, we refer to [10-13, 16, 26-28].

\footnotetext{
${ }^{*}$ Corresponding author

Email address: vakhanmaths@gmail .com (Vakeel A. Khan)

doi: $10.22436 /$ jmcs.020.03.05
}

Received: 2019-04-16 Revised: 2019-10-11 Accepted: 2019-10-31 
Throughout the paper, by $\ell_{\infty}, c$, and $c_{0}$ we denote the spaces of all bounded, convergent, and null sequences, respectively. Let $A=\left(a_{n k}\right)$ be an infinite matrix of real or complex numbers $a_{n k}$, where $n, k \in \mathbb{N}$ and let $x=\left(x_{k}\right) \in \omega$. Consider the new sequence

$$
A_{n}(x)=\sum_{k=0}^{\infty} a_{n k} x_{k}, \quad \text { for each } n \in \mathbb{N},
$$

that is, the sequence obtained on transforming the sequence $x$ by the matrix $A$. Assume that the series on the right hand side of (1.1) converges for each $n \in \mathbb{N}$. Then the transformation $A$ applies to the sequence $x$, and the sequence $A_{n}(x)$ is called the A-transform of $x$. The necessary and sufficient conditions for those matrices $A$ that maps convergent sequence into another convergent sequence were given by well known Kojima-Schur Theorem 4.1,I (see, [4], p. 63) as follows:

(i) $\sum_{k=1}^{\infty}\left|a_{n k}\right| \leqslant M$ for every $n>n_{0}$;

(ii) $\lim _{n \rightarrow \infty} a_{n k}=\alpha_{k}$ for every fixed $k$;

(iii) $\sum_{k=1}^{\infty} a_{n k}=A_{n} \rightarrow \alpha$ as $n \rightarrow \infty$.

An infinite matrix $A=\left(a_{n k}\right)$ is called a triangle if $a_{n k}=0$ for $k>n$ and $a_{n n} \neq 0$ for all $n \in \mathbb{N}$. Also a triangle matrix $A=\left(a_{n k}\right)$ uniquely has an inverse $A^{-1}$ which is a triangle matrix. Let $\lambda$ be an arbitrary sequence space. Then for every sequence $x=\left(x_{k}\right) \in \lambda$, the matrix domain of an infinite matrix $A$ in a sequence space $\lambda$ is a sequence space defined by

$$
\lambda_{A}:=\left\{x=\left(x_{k}\right) \in \omega: A x \in \lambda\right\} .
$$

Motivated by various properties of matrix domains of triangles such as, if $A$ is a triangle and $\lambda$ is a BKspace, then $\lambda_{A}$ is also a BK-space with the norm given by $\|x\|_{\lambda_{A}}=\|A x\|_{\lambda}$ for all $x \in \lambda_{A}$ (see [2, Theorem 8.1.4]), the study of such matrices attracted the attention of many researchers to dig deeper in this area, for instance [1, 3, 19-21], and the references therein.

Recall in [9] the Hilbert matrix is an infinite matrix $H=\left(a_{n k}\right)$ whose entries are $a_{n k}=(n+k-1)^{-1}$ for $n, k \in \mathbb{N}$. The Hilbert matrix $H$ was used in the theory of sequence spaces and considered as a bounded linear operator on the spaces of all $p$-summable sequences $\ell_{p}$ with norm $\|H\|_{p}=\frac{\pi}{\sin (\pi / p)}$ for $1<p<\infty$ (see, [8]). Also, the Hilbert matrix can be viewed as an operator on spaces of analytic functions by its action on Taylor coefficients in [5]. Recently, by using the square Hilbert matrix $\mathrm{H}$ of order $n$, Polat [22] has introduced some new sequence spaces $h_{\infty}, h_{c}$ and $h_{0}$ as the sets of all sequences whose $\mathrm{H}$-transforms of the sequence $x=\left(x_{k}\right) \in \omega$ are in the spaces $\ell_{\infty}, c$ and $c_{0}$, respectively, that is

$$
\lambda_{H}=\left\{x=\left(x_{k}\right) \in \omega:\left(\sum_{k=1}^{m} \frac{x_{k}}{n+k-1}\right) \in \lambda\right\} \quad \text { for } \lambda \in\left\{c_{0}, c, \ell_{\infty}\right\} .
$$

Throughout the paper $c_{0}^{I}, c^{I}$ and $\ell_{\infty}^{I}$, denote the spaces of all I-null, I-convergent, and I-bounded sequences, respectively. In this paper, by using the triangle Hilbert matrix $H=\left(a_{n k}\right)$ defined by

$$
a_{n k}= \begin{cases}\frac{1}{n+k-1}, & \text { if } 1 \leqslant k \leqslant n, \\ 0, & \text { if } k>n\end{cases}
$$

for all $n, k \in \mathbb{N}$, one can easily check that the conditions (i), (ii), and (iii) hold for the triangle Hilbert matrix $H$, and the notion of ideal convergence we define new sequence spaces $c_{0}^{I}(H), c^{I}(H), \ell_{\infty}^{I}(H)$ and $\ell_{\infty}(H)$ as the sets of all sequences whose $H$-transforms are in the spaces $c_{0}^{I}, c^{I}, \ell_{\infty}^{I}$ and $\ell_{\infty}$, respectively. We define the sequence $H_{n}(x)$ that will be frequently used, as $H$-transform of the sequence $x=\left(x_{k}\right) \in \omega$, as follows:

$$
H_{n}(x)=\sum_{k=1}^{n} \frac{x_{k}}{n+k-1} \text { for } n, k \in \mathbb{N} .
$$

Further, we study some topological and algebraic properties and present some inclusion relations on these results. 
Now, we recall some definitions and lemmas which will be used throughout the paper.

Definition 1.1 ([25]). If $K=\{k \in K: k \leqslant n\}$ is a subset of $\mathbb{N}$, then the natural density of the set $K$ is given by

$$
\mathrm{d}(\mathrm{K})=\lim _{\mathrm{n} \rightarrow \infty} \frac{1}{\mathrm{n}}|\mathrm{K}|
$$

whenever the limit exists, here $|\mathrm{B}|$ denotes the cardinality of the set $\mathrm{B}$.

Definition 1.2 ([6]). A sequence $x=\left(x_{k}\right) \in \omega$ is said to be statistically convergent to a number $\ell \in \mathbb{R}$ if, for every $\epsilon>0, d\left(\left\{k \in \mathbb{N}:\left|x_{k}-\ell\right| \geqslant \epsilon\right\}\right)=0$. And we write st-lim $x_{k}=\ell$. In case, $\ell=0$ then $x=\left(x_{k}\right) \in \omega$ is said to be st-null.

Definition $1.3([23])$. A sequence $x=\left(x_{k}\right) \in \omega$ is said to be I-Cauchy if, for every $\epsilon>0$, there exists a number $N=N(\epsilon) \in \mathbb{N}$ such that $\left\{k \in \mathbb{N}:\left|x_{k}-x_{N}\right| \geqslant \epsilon\right\} \in I$.

Definition 1.4 ([18]). A sequence $x=\left(x_{k}\right) \in \omega$ is said to be I-convergent to a number $\ell \in \mathbb{R}$ if, for every $\epsilon>0,\left\{k \in \mathbb{N}:\left|x_{k}-\ell\right| \geqslant \epsilon\right\} \in \mathrm{I}$. And we write $\mathrm{I}-\lim x_{k}=\ell$. In case, $\ell=0$ then $\left(x_{k}\right) \in \omega$ is said to be I-null.

Definition 1.5 ([14]). A sequence $x=\left(x_{k}\right) \in \omega$ is said to be I-bounded if there exists $K>0$, such that $\left\{k \in \mathbb{N}:\left|x_{k}\right|>K\right\} \in I$.

Definition $1.6([23])$. Let $x=\left(x_{k}\right)$ and $z=\left(z_{k}\right)$ be two sequences. We say that $x_{k}=z_{k}$ for almost all $k$ relative to I (in short a.a.k.r.I) if $\left\{k \in \mathbb{N}: x_{k} \neq z_{k}\right\} \in I$.

Definition 1.7 ([23]). A sequence space $E$ is said to be solid or normal, if $\left(\alpha_{k} x_{k}\right) \in E$ whenever $\left(x_{k}\right) \in E$ and for any sequence of scalars $\left(\alpha_{k}\right) \in \omega$ with $\left|\alpha_{k}\right|<1$, for every $k \in \mathbb{N}$.

Definition 1.8 ([23]). Let $K=\left\{k_{i} \in \mathbb{N}: k_{1}<k_{2}<\cdots\right\} \subseteq \mathbb{N}$ and $E$ be a sequence space. A $K$-step space of $E$ is a sequence space

$$
\lambda_{\mathrm{K}}^{\mathrm{E}}=\left\{\left(x_{\mathrm{k}_{\mathrm{i}}}\right) \in \omega:\left(x_{\mathrm{k}}\right) \in \mathrm{E}\right\} .
$$

A canonical pre-image of a sequence $\left(x_{k_{i}}\right) \in \lambda_{K}^{E}$ is a sequence $\left(y_{k}\right) \in \omega$ defined as follows:

$$
y_{k}= \begin{cases}x_{k}, & \text { if } k \in K, \\ 0, & \text { otherwise. }\end{cases}
$$

A canonical pre-image of a step space $\lambda_{\mathrm{K}}^{\mathrm{E}}$ is a set of canonical pre-images of all elements in $\lambda_{\mathrm{K}}^{\mathrm{E}}$, i.e., $y$ is in canonical pre-image of $\lambda_{\mathrm{K}}^{\mathrm{E}}$ iff $\mathrm{y}$ is canonical pre-image of some element $x \in \lambda_{\mathrm{K}}^{\mathrm{E}}$.

Definition 1.9 ([23]). A sequence space $E$ is said to be monotone, if it contains the canonical pre-images of its step space.

Lemma 1.10 ([23]). Every solid space is monotone.

Lemma 1.11 ([24]). Let $\mathrm{K} \in \mathcal{F}(\mathrm{I})$ and $\mathrm{M} \subseteq \mathbb{N}$. If $\mathrm{M} \notin \mathrm{I}$, then $\mathrm{M} \cap \mathrm{K} \notin \mathrm{I}$.

\section{Main results}

In this section, we define new sequence spaces $c_{0}^{\mathrm{I}}(\mathrm{H}), \mathrm{c}^{\mathrm{I}}(\mathrm{H}), \ell_{\infty}^{\mathrm{I}}(\mathrm{H})$, and $\ell_{\infty}(\mathrm{H})$ as the sets of all sequences whose $\mathrm{H}$-transforms are in the spaces $c_{0}^{\mathrm{I}}, c^{\mathrm{I}}, \ell_{\infty}^{\mathrm{I}}$, and $\ell_{\infty}$, respectively. Further, we study some topological and algebraic properties and present some inclusion relations on these resulting. Throughout the paper, we suppose that the sequence $x=\left(x_{k}\right) \in \omega$ and $H_{n}(x)$ are connected with the relation (1.3) and I is an admissible ideal of subset of $\mathbb{N}$.

$$
c_{0}^{\mathrm{I}}(\mathrm{H}):=\left\{x=\left(x_{\mathrm{k}}\right) \in \omega:\left\{\mathrm{n} \in \mathbb{N}:\left|\mathrm{H}_{\mathrm{n}}(x)\right| \geqslant \epsilon\right\} \in \mathrm{I}\right\},
$$




$$
\begin{aligned}
c^{I}(H) & :=\left\{x=\left(x_{k}\right) \in \omega:\left\{n \in \mathbb{N}:\left|H_{n}(x)-\ell\right| \geqslant \epsilon, \text { for some } \ell \in \mathbb{R}\right\} \in I\right\} \\
\ell_{\infty}^{I}(H) & :=\left\{x=\left(x_{k}\right) \in \omega: \exists K>0 \text { s.t }\left\{n \in \mathbb{N}:\left|H_{n}(x)\right| \geqslant K\right\} \in I\right\} \\
\ell_{\infty}(H) & :=\left\{x=\left(x_{k}\right) \in \omega: \sup _{n}\left|H_{n}(x)\right|<\infty\right\} .
\end{aligned}
$$

We write

$$
\mathrm{m}_{0}^{\mathrm{I}}(\mathrm{H}):=\mathrm{c}_{0}^{\mathrm{I}}(\mathrm{H}) \cap \ell_{\infty}(\mathrm{H}), \quad \text { and } \quad \mathrm{m}^{\mathrm{I}}(\mathrm{H}):=\mathrm{c}^{\mathrm{I}}(\mathrm{H}) \cap \ell_{\infty}(\mathrm{H}) .
$$

With the notation of (1.2), the sequence spaces $c_{0}^{\mathrm{I}}(\mathrm{H}), \mathrm{c}^{\mathrm{I}}(\mathrm{H}), \ell_{\infty}^{\mathrm{I}}(\mathrm{H}), \mathrm{m}^{\mathrm{I}}(\mathrm{H})$, and $\mathrm{m}_{0}^{\mathrm{I}}(\mathrm{H})$ can be redefined as follows:

$$
c_{0}^{\mathrm{I}}(\mathrm{H})=\left(\mathrm{c}_{0}^{\mathrm{I}}\right)_{\mathrm{H}}, \quad \mathrm{c}^{\mathrm{I}}(\mathrm{H})=\left(\mathrm{c}^{\mathrm{I}}\right)_{\mathrm{H}}, \quad \ell_{\infty}^{\mathrm{I}}(\mathrm{H})=\left(\ell_{\infty}^{\mathrm{I}}\right)_{\mathrm{H}}, \quad \mathrm{m}^{\mathrm{I}}(\mathrm{H})=\left(\mathrm{m}^{\mathrm{I}}\right)_{\mathrm{H}}, \quad \text { and } \quad \mathrm{m}_{0}^{\mathrm{I}}(\mathrm{H})=\left(\mathrm{m}_{0}^{\mathrm{I}}\right)_{\mathrm{H}} .
$$

Definition 2.1. Let $I$ is an admissible ideal of subset of $\mathbb{N}$. If for each $\epsilon>0$ there exists a number $N=N(\epsilon) \in \mathbb{N}$ such that $\left\{n \in \mathbb{N}:\left|H_{n}(x)-H_{N}(x)\right| \geqslant \epsilon\right\} \in I$ then a sequence $x=\left(x_{k}\right) \in \omega$ is called Hilbert I-Cauchy.

Example 2.1. Define $I_{f}=\{A \subseteq \mathbb{N}: A$ is finite $\}$. $I_{f}$ is an admissible ideal in $\mathbb{N}$ and $c^{I_{f}}(H)=h_{c}$.

Example 2.2. Define a non-trivial ideal as $I_{d}=\{A \subseteq \mathbb{N}: d(A)=0\}$, where $d(A)$ is the natural density of the set $A$. In this case $c^{I_{d}}(H)=S(H)$. Where we define $S(H)$ as the space of all Hilbert statistically convergent sequences as follows:

$$
S(H):=\left\{x=\left(x_{k}\right) \in \omega: d\left(\left\{n \in \mathbb{N}:\left|H_{n}(x)-\ell\right| \geqslant \epsilon\right\}\right)=0, \text { for some } \ell \in \mathbb{R}\right\} .
$$

Remark 2.2. Hilbert convergent sequence is obviously Hilbert statistically convergent since all finite subsets of the natural numbers have density zero. However, the converse is not true. For example, define the sequence $x=\left(x_{k}\right) \in \omega$ such that

$$
H_{n}(x)= \begin{cases}1, & \text { if } n \text { is a square, } \\ 0, & \text { otherwise, }\end{cases}
$$

that is, $H_{n}(x)=\{1,0,0,1,0,0,0,0,1,0, \ldots\}$ and let $\ell=0$. Then,

$$
\left\{n \in \mathbb{N}:\left|H_{n}(x)-\ell\right| \geqslant \epsilon\right\} \subset\left\{1,4,9,16, \ldots, i^{2}, \ldots\right\} .
$$

Since the set of squares of natural numbers has natural density zero, we have

$$
\mathrm{d}\left(\left\{\mathrm{n} \in \mathbb{N}:\left|\mathrm{H}_{\mathrm{n}}(x)-\ell\right| \geqslant \epsilon\right\}\right)=0 .
$$

This implies that the sequence $\left(x_{k}\right) \in S(H)$, but $\left(x_{k}\right) \notin h_{c}$.

Theorem 2.3. The sequence spaces $c^{\mathrm{I}}(\mathrm{H}), \mathrm{c}_{0}^{\mathrm{I}}(\mathrm{H}), \ell_{\infty}^{\mathrm{I}}(\mathrm{H}), \mathrm{m}_{0}^{\mathrm{I}}(\mathrm{H})$, and $\mathrm{m}^{\mathrm{I}}(\mathrm{H})$ are linear over $\mathbb{R}$.

Proof. Let $x=\left(x_{k}\right), y=\left(y_{k}\right)$ be two arbitrary elements of the space $c^{I}(H)$ and $\alpha, \beta$ are scalars. Now, since $x, y \in c^{I}(H)$, then for given $\epsilon>0$, there exist $\ell_{1}, \ell_{2} \in \mathbb{R}$, such that

$$
\left\{n \in \mathbb{N}:\left|H_{n}(x)-\ell_{1}\right| \geqslant \frac{\epsilon}{2}\right\} \in I \quad \text { and } \quad\left\{n \in \mathbb{N}:\left|H_{n}(y)-\ell_{2}\right| \geqslant \frac{\epsilon}{2}\right\} \in I .
$$

Now, let

$$
A_{1}=\left\{n \in \mathbb{N}:\left|H_{n}(x)-\ell_{1}\right|<\frac{\epsilon}{2|\alpha|}\right\} \in \mathcal{F}(\mathrm{I}), \quad A_{2}=\left\{n \in \mathbb{N}:\left|H_{n}(y)-\ell_{2}\right|<\frac{\epsilon}{2|\beta|}\right\} \in \mathcal{F}(\mathrm{I}),
$$


be such that $A_{1}^{c}, A_{2}^{c} \in I$. Then

$$
\begin{aligned}
A_{3} & =\left\{n \in \mathbb{N}:\left|H_{n}(\alpha x+\beta y)-\left(\alpha \ell_{1}+\beta \ell_{2}\right)\right|<\epsilon\right\} \\
& \supseteq\left\{\left\{n \in \mathbb{N}:\left|H_{n}(x)-\ell_{1}\right|<\frac{\epsilon}{2|\alpha|}\right\} \cap\left\{n \in \mathbb{N}:\left|H_{n}(y)-\ell_{2}\right|<\frac{\epsilon}{2|\beta|}\right\}\right\} .
\end{aligned}
$$

Thus, the sets on right hand-side of equation (2.1) belong to $\mathcal{F}(\mathrm{I})$. By definition of filter associated with ideal, the complement of the set on left-hand side of (2.1) belongs to I. This implies that $(\alpha x+\beta y) \in c^{\mathrm{I}}(\mathrm{H})$. Hence, $c^{I}(H)$ is linear space. The proof of the remaining results is similar.

Theorem 2.4. The spaces $\mathrm{X}(\mathrm{H})$ are normed spaces with the norm

$$
\|x\|_{X(H)}=\sup _{n}\left|H_{n}(x)\right|, \quad \text { where } X \in\left\{c^{I}, c_{0}^{I}, \ell_{\infty}^{I}, \ell_{\infty}\right\} .
$$

Proof. The proof of the result is easy in view of existing techniques and hence omitted.

Theorem 2.5. A sequence $\mathrm{x}=\left(\mathrm{x}_{\mathrm{k}}\right) \in \omega$ is Hilbert I-convergent if and only if for every $\epsilon>0$, there exists $\mathrm{N}=\mathrm{N}(\epsilon) \in \mathbb{N}$, such that

$$
\left\{n \in \mathbb{N}:\left|H_{n}(x)-H_{N}(x)\right|<\epsilon\right\} \in \mathcal{F}(I) .
$$

Proof. Suppose that the sequence $x=\left(x_{k}\right) \in \omega$ is Hilbert I-convergent to some number $\ell \in \mathbb{R}$, then for a given $\epsilon>0$, we have

$$
A_{\epsilon}=\left\{n \in \mathbb{N}:\left|H_{n}(x)-\ell\right|<\frac{\epsilon}{2}\right\} \in \mathcal{F}(I) .
$$

Fix an integer $N=N(\epsilon) \in A_{\epsilon}$. Then, we have

$$
\left|H_{n}(x)-H_{N}(x)\right| \leqslant\left|H_{n}(x)-\ell\right|+\left|\ell-H_{N}(x)\right|<\frac{\epsilon}{2}+\frac{\epsilon}{2}=\epsilon
$$

for all $n \in A_{\epsilon}$. Hence, (2.3) holds.

Conversely, suppose that (2.3) holds for all $\epsilon>0$. Then,

$$
B_{\epsilon}=\left\{n \in \mathbb{N}: H_{n}(x) \in\left[H_{n}(x)-\epsilon, H_{n}(x)+\epsilon\right]\right\} \in \mathcal{F}(I), \text { for all } \epsilon>0 .
$$

Let $J_{\epsilon}=\left[H_{n}(x)-\epsilon, H_{n}(x)+\epsilon\right]$. Fixing $\epsilon>0$, we have $B_{\epsilon} \in \mathcal{F}(I)$ and $B_{\frac{\epsilon}{2}} \in \mathcal{F}(I)$. Hence, $B_{\epsilon} \cap B_{\frac{\epsilon}{2}} \in \mathcal{F}(I)$. This implies that

$$
\mathrm{J}=\mathrm{J}_{\epsilon} \cap \mathrm{J}_{\frac{\epsilon}{2}} \neq \emptyset
$$

that is,

$$
\left\{\mathrm{n} \in \mathbb{N}: \mathrm{H}_{\mathrm{n}}(\mathrm{x}) \in \mathrm{J}\right\} \in \mathcal{F}(\mathrm{I})
$$

and thus

$$
\operatorname{diam}(\mathrm{J}) \leqslant \frac{1}{2} \operatorname{diam}\left(\mathrm{J}_{\epsilon}\right),
$$

where the diam of $J$ denotes the length of an interval J. Proceeding in this way, by induction we get a sequence of closed intervals $\mathrm{J}_{\epsilon}=\mathrm{I}_{0} \supseteq \mathrm{I}_{1} \supseteq \cdots \supseteq \mathrm{I}_{\mathrm{n}} \supseteq \cdots$ such that

$$
\operatorname{diam}\left(I_{n}\right) \leqslant \frac{1}{2} \operatorname{diam}\left(I_{n-1}\right), \text { for } n=(2,3, \ldots)
$$

and

$$
\left\{n \in \mathbb{N}: H_{n}(x) \in I_{n}\right\} \in \mathcal{F}(\mathrm{I}) .
$$

Then, there exists a number $\ell \in \bigcap_{n \in \mathbb{N}} I_{n}$ and it is a routine work to verify that $\ell=I-\lim H_{n}(x)$ showing that $x=\left(x_{k}\right) \in \omega$ is Hilbert I-convergent. Hence the result. 
Theorem 2.6. The inclusions $\mathrm{c}_{0}^{\mathrm{I}}(\mathrm{H}) \subset \mathrm{c}^{\mathrm{I}}(\mathrm{H}) \subset \ell_{\infty}^{\mathrm{I}}(\mathrm{H})$ are strict.

Proof. The inclusion $\mathrm{c}_{0}^{\mathrm{I}}(\mathrm{H}) \subset \mathrm{c}^{\mathrm{I}}(\mathrm{H})$ is obvious. Now, to show its strictness, consider the sequence $x=$ $\left(x_{k}\right) \in \omega$ such that $H_{n}(x)=2$. It easy to see that the sequence $H_{n}(x) \in c^{I}$ but $H_{n}(x) \notin c_{0}^{I}$, that is, $x \in c^{I}(H) \backslash c_{0}^{I}(H)$. Next, let $x=\left(x_{k}\right) \in c^{I}(H)$. Then there exists $\ell \in \mathbb{R}$ such that $I-\lim H_{n}(x)=\ell$, that is,

$$
\left\{n \in \mathbb{N}:\left|H_{n}(x)-\ell\right| \geqslant \epsilon\right\} \in I .
$$

We have

$$
\left|H_{n}(x)\right|=\left|H_{n}(x)-\ell+\ell\right| \leqslant\left|H_{n}(x)-\ell\right|+|\ell| .
$$

From this it easily follows that the sequence $\left(x_{k}\right)$ must belongs to $\ell_{\infty}^{I}(H)$. Further, we show the strictness of the inclusion $\mathrm{c}^{\mathrm{I}}(\mathrm{H}) \subset \ell_{\infty}^{\mathrm{I}}(\mathrm{H})$ by constructing the following example.

Example 2.3. Consider the sequence $x=\left(x_{k}\right) \in \omega$ such that

$$
H_{n}(x)= \begin{cases}\sqrt{n}, & \text { if } n \text { is square, } \\ 1, & \text { if } n \text { is odd non-square, } \\ 0, & \text { if } n \text { is even non-square. }\end{cases}
$$

Then, the sequence $H_{n}(x) \in \ell_{\infty}^{I}$, but $H_{n}(x) \notin c^{I}$ which implies that the sequence $x \in \ell_{\infty}^{I}(H) \backslash c^{I}(H)$.

Thus, the inclusions $\mathrm{c}_{0}^{\mathrm{I}}(\mathrm{H}) \subset \mathrm{c}^{\mathrm{I}}(\mathrm{H}) \subset \ell_{\infty}^{\mathrm{I}}(\mathrm{H})$ is strict.

Remark 2.7. Hilbert bounded sequence is obviously Hilbert I-bounded as the empty set belongs to the ideal I. However, the converse is not true. For example, consider the sequence $x=\left(x_{k}\right) \in \omega$ such that

$$
H_{n}(x)= \begin{cases}\frac{n^{2}}{n+1}, & \text { if } n \text { is prime, } \\ 0, & \text { otherwise. }\end{cases}
$$

Clearly $H_{n}(x)$ is not a bounded sequence. However, $\left\{n \in \mathbb{N}:\left|H_{n}(x)\right|>1\right\} \in I$. Hence, $\left(x_{k}\right)$ is Hilbert I-bounded.

Theorem 2.8. The sequence spaces:

(i) $\mathrm{c}^{\mathrm{I}}(\mathrm{H})$ and $\ell_{\infty}(\mathrm{H})$, overlap but neither one contains the other;

(ii) $\mathrm{c}_{0}^{\mathrm{I}}(\mathrm{H})$ and $\ell_{\infty}(\mathrm{H})$, overlap but neither one contains the other.

Proof.

(i) We prove that $c^{I}(H)$ and $\ell_{\infty}(H)$ are not disjoint. Consider the sequence $x=\left(x_{k}\right) \in \omega$ such that $H_{n}(x)=\frac{1}{n}$ for $n \in \mathbb{N}$. Then, $x \in c^{I}(H)$ but $x \in \ell_{\infty}(H)$. Next, define the sequence $x=\left(x_{k}\right) \in \omega$ such that

$$
H_{n}(x)= \begin{cases}\sqrt{n}, & \text { if } n \text { is square, } \\ 0, & \text { otherwise }\end{cases}
$$

Thus, $x \in c^{I}(H)$ but $x \notin \ell_{\infty}(H)$. Next, choose the sequence $x=\left(x_{k}\right) \in \omega$ such that

$$
H_{n}(x)= \begin{cases}n, & \text { if is even, } \\ 0, & \text { otherwise }\end{cases}
$$

Then $(x) \in \ell_{\infty}(H)$ but $x \notin c^{I}(H)$.

(ii) The proof is similar to the proof of part one.

Theorem 2.9. The spaces $m^{\mathrm{I}}(\mathrm{H})$ and $\mathrm{m}_{0}^{\mathrm{I}}(\mathrm{H})$ are closed subspace of $\ell_{\infty}(\mathrm{H})$. 
Proof. Let $\left(x_{k}^{(i)}\right)$ be a Cauchy sequence in $m^{I}(H) \subset \ell_{\infty}(H)$. Then, $\left(x_{k}^{(i)}\right)$ converges in $\ell_{\infty}(H)$ and we have $\lim _{i \rightarrow \infty} H_{n}^{(i)}(x)=H_{n}(x)$. Let $I-\lim H_{n}^{(i)}(x)=\ell_{i}$ for each $i \in \mathbb{N}$. Then, we have to show that

(i) $\left(\ell_{i}\right)$ is convergent say to $\ell_{\text {; }}$

(ii) $\mathrm{I}-\lim \mathrm{H}_{\mathrm{n}}(\mathrm{x})=\ell$.

(i) Since $\left(x_{k}^{(i)}\right)$ is a Cauchy sequence, for each $\epsilon>0$ there exists $n_{0} \in \mathbb{N}$ such that

$$
\left|H_{n}^{(i)}(x)-H_{n}^{(j)}(x)\right|<\frac{\epsilon}{3}, \text { for all } i, j \geqslant n_{0} .
$$

Now let $A_{i}$ and $A_{j}$ be the following sets in I:

$$
A_{i}=\left\{n \in \mathbb{N}:\left|H_{n}^{(i)}(x)-\ell_{i}\right| \geqslant \frac{\epsilon}{3}\right\}
$$

and

$$
A_{j}=\left\{n \in \mathbb{N}:\left|H_{n}^{(j)}(x)-\ell_{j}\right| \geqslant \frac{\epsilon}{3}\right\} .
$$

Consider $i, j \geqslant n_{0}$ and $n \notin A_{i} \cap A_{j}$. Then we have

$$
\left|\ell_{i}-\ell_{j}\right| \leqslant\left|H_{n}^{(i)}(x)-\ell_{i}\right|+\left|H_{n}^{(j)}(x)-\ell_{j}\right|+\left|H_{n}^{(i)}(x)-H_{n}^{(j)}(x)\right|<\epsilon
$$

by (2.4), (2.5), and (2.6). Thus, $\left(\ell_{i}\right)$ is a Cauchy sequence in $\mathbb{R}$ and thus convergent say to $\ell$, that is, $\lim _{i \rightarrow \infty} \ell_{i}=\ell$.

(ii) Let $\delta>0$ be given, then we can find $m_{0}$ such that

$$
\left|\ell_{i}-\ell\right|<\frac{\delta}{3}, \text { for each } i>m_{0} \text {. }
$$

We have $\left(x_{k}^{(i)}\right) \rightarrow x_{k}$ as $i \rightarrow \infty$. Thus

$$
\left|H_{n}^{(i)}(x)-H_{n}(x)\right|<\frac{\delta}{3}, \text { for each } i>m_{0} .
$$

Since $\left(H_{n}^{(j)}(x)\right)$ is I-convergent to $\ell_{j}$, there exists $D \in I$ such that for each $n \notin D$, we have

$$
\left|H_{n}^{(j)}(x)-\ell_{j}\right|<\frac{\delta}{3}
$$

Without loss of generality, let $j>m_{0}$ then for all $n \notin D$, we have by (2.7), (2.8), and (2.9) that

$$
\left|H_{n}(x)-\ell\right| \leqslant\left|H_{n}(x)-H_{n}^{(j)}(x)\right|+\left|H_{n}^{(j)}(x)-\ell_{j}\right|+\left|\ell_{j}-\ell\right|<\delta .
$$

Hence $\left(x_{k}\right)$ is Hilbert I-convergent to $\ell$. Thus $m^{I}(H)$ is a closed subspace of $\ell_{\infty}(H)$. Similarly the other cases can be established.

Theorem 2.10. The sequence spaces $\mathrm{c}^{\mathrm{I}}(\mathrm{H}), \mathrm{c}_{0}^{\mathrm{I}}(\mathrm{H})$, and $\ell_{\infty}^{\mathrm{I}}(\mathrm{H})$ are BK-spaces according to their norms defined by (2.2).

Proof. We know that the sequence spaces $\mathrm{c}^{\mathrm{I}}, \mathrm{c}_{0}^{\mathrm{I}}$, and $\ell_{\infty}^{\mathrm{I}}$ are BK-spaces with their sup-norm. Furthermore, (1.2) holds and the Hilbert matrix is a triangle matrix. By taking into account these three facts and Theorem of Wilansky [29], we conclude that the sequence spaces are BK-spaces. This completes the proof of the theorem.

Since the spaces $\mathrm{m}^{\mathrm{I}}(\mathrm{H}) \subset \ell_{\infty}(\mathrm{H})$ and $\mathrm{m}_{0}^{\mathrm{I}}(\mathrm{H}) \subset \ell_{\infty}(\mathrm{H})$ are strict, in view of Theorem 2.9, we have the following result. 
Theorem 2.11. The spaces $\mathrm{m}^{\mathrm{I}}(\mathrm{H})$ and $\mathrm{m}_{0}^{\mathrm{I}}(\mathrm{H})$ are nowhere dense subset of $\ell_{\infty}(\mathrm{H})$.

Theorem 2.12. The spaces $\mathrm{c}_{0}^{\mathrm{I}}(\mathrm{H})$ and $\mathrm{m}_{0}^{\mathrm{I}}(\mathrm{H})$ are solid and monotone.

Proof. We will prove the result for $c_{0}^{\mathrm{I}}(\mathrm{H})$ and for $\mathrm{m}_{0}^{\mathrm{I}}(\mathrm{H})$ the result can be established similarly. Let $x=\left(x_{k}\right) \in c_{0}^{I}(H)$. For $\epsilon>0$, the set

$$
\left\{\mathrm{n} \in \mathbb{N}:\left|\mathrm{H}_{\mathrm{n}}(\mathrm{x})\right| \geqslant \epsilon\right\}
$$

belongs to I. Let $\alpha=\left(\alpha_{k}\right)$ be a sequence of scalars with $|\alpha| \leqslant 1$ for all $k \in \mathbb{N}$. Then,

$$
\left|H_{n}(\alpha x)\right|=\left|\alpha H_{n}(x)\right| \leqslant|\alpha|\left|H_{n}(x)\right| \leqslant\left|H_{n}(x)\right|, \text { for all } n \in \mathbb{N} \text {. }
$$

From this inequality and from (2.10) we have

$$
\left\{n \in \mathbb{N}:\left|H_{n}(\alpha x)\right| \geqslant \epsilon\right\} \subseteq\left\{n \in \mathbb{N}:\left|H_{n}(x)\right| \geqslant \epsilon\right\} \in I
$$

implies that

$$
\left\{n \in \mathbb{N}:\left|H_{n}(\alpha x)\right| \geqslant \epsilon\right\} \in I .
$$

Therefore, $\left(\alpha x_{k}\right) \in c_{0}^{I}(H)$. Hence, the space $c_{0}^{I}(H)$ is solid, and hence by Lemma 1.10 the space $c_{0}^{\mathrm{I}}(H)$ is monotone.

Theorem 2.13. If $\mathrm{I}$ is neither maximal nor $\mathrm{I}=\mathrm{I}_{\mathrm{f}}$, then the spaces $\mathrm{c}^{\mathrm{I}}(\mathrm{H})$ and $\mathrm{m}^{\mathrm{I}}(\mathrm{H})$ are neither monotone nor solid.

Proof. We prove this result with the help of the following example.

Example 2.4. Let $I=I_{f}$. Let $K=\{n \in \mathbb{N}: n$ is odd $\}$. Consider the $K$-step space $E_{K}$ of $E$ defined as:

$$
E_{K}=\left\{\left(x_{k}\right) \in \omega:\left(x_{k}\right) \in E\right\} .
$$

Define the sequence $\left(y_{k}\right) \in E_{K}$ such that

$$
H_{n}(y)= \begin{cases}H_{n}(x), & \text { if } n \in K, \\ 0, & \text { otherwise. }\end{cases}
$$

Consider the sequence $\left(x_{k}\right)$ such that $H_{n}(x)=3$, for all $n \in \mathbb{N}$. Then, $\left(x_{k}\right) \in E(H)$, but its K-step space pre-image does not belongs to $E(H)$, where $E=c^{I}$ and $m^{I}$. Thus, $E(H)$ are not monotone. Hence, by Lemma 1.10 the spaces $E(H)$ are not solid.

Theorem 2.14. Let $\mathrm{x}=\left(\mathrm{x}_{\mathrm{k}}\right) \in \mathrm{\omega}$ and let $\mathrm{I}$ be a non-trivial admissible ideal in $\mathbb{N}$. If there is a sequence $\mathrm{y}=\left(\mathrm{y}_{\mathrm{k}}\right) \in \mathrm{c}^{\mathrm{I}}(\mathrm{H})$ such that $\mathrm{H}_{\mathrm{n}}(\mathrm{x})=\mathrm{H}_{\mathrm{n}}(\mathrm{y})$ for almost all $\mathrm{n}$ relative to $\mathrm{I}$, then $\mathrm{x} \in \mathrm{c}^{\mathrm{I}}(\mathrm{H})$.

Proof. Suppose that $H_{n}(x)=H_{n}(y)$ for almost all $n$ relative to $I$, that is

$$
\left\{\mathrm{n} \in \mathbb{N}: \mathrm{H}_{\mathrm{n}}(\mathrm{x}) \neq \mathrm{H}_{\mathrm{n}}(\mathrm{y})\right\} \in \mathrm{I} .
$$

And let $\left(y_{k}\right)$ be a sequence which is Hilbert I-convergent to $\ell$. Then, for every $\epsilon>0$, we have

$$
\left\{\mathrm{n} \in \mathbb{N}:\left|\mathrm{H}_{\mathrm{n}}(\mathrm{y})-\ell\right| \geqslant \epsilon\right\} \in \mathrm{I} .
$$

Since I is an admissible ideal, then the result follows from the following inclusion

$$
\left\{n \in \mathbb{N}:\left|H_{n}(x)-\ell\right| \geqslant \epsilon\right\} \subseteq\left\{n \in \mathbb{N}: H_{n}(x) \neq H_{n}(y)\right\} \cup\left\{n \in \mathbb{N}:\left|H_{n}(y)-\ell\right| \geqslant \epsilon\right\} .
$$




\section{Acknowledgment}

The authors would like to thank the referees and the editor for their careful reading and their valuable comments.

\section{References}

[1] F. Başar, B. Altay, On the space of sequences of $\mathrm{p}$-bounded variation and related matrix mappings, Ukrainian Math. J., 55 (2003), 136-147. 1

[2] J. Boos, F. Cass, Classical and modern methods in summability, Oxford University Press, Oxford, (2000). 1

[3] M. Candan, Domain of the double sequential band matrix in the spaces of convergent and null sequences, Adv. Difference Equ., 2014 (2014), 18 pages. 1

[4] R. G. Cooke, Infinite matrices and sequence spaces, Dover, New York, (2014). 1

[5] E. Diamantopoulos, A. G. Siskakis, Composition operators and the Hilbert matrix, Studia Math., 140 (2000), 191-198. 1

[6] H. Fast, Sur la convergence statistique, (French) Colloq. Math., 2 (1951), 241-244. 1, 1.2

[7] R. Filipów, J. Tryba, Ideal convergence versus matrix summability, Stud. Math., 245 (2019), 101-127. 1

[8] G. H. Hardy, J. E. Littlewood, G. Polya, Inequalities, Cambridge University Press, Cambridge, (1934). 1

[9] D. Hilbert, Ein beitrag zur theorie des legendre'schen polynoms, (German) Acta Math., 18 (1894), 155-159. 1

[10] M. Jeyaram Bharathi, S. Velmurugan, N. Subramanian, R. Srikanth, On Triple sequence space of Bernstein operator of rough $\mathrm{I}_{\lambda}$-statistical convergence of weighted $\mathrm{g}(\mathrm{A})$, J. Intel. Fuzzy Syst., 36 (2019), 13-27. 1

[11] M. Kemal Ozdemir, A. Esi, N. Subramanian, Rough convergence of Bernstein fuzzy I-convergent of $\chi_{f}^{3 \mathrm{FI}}$ and $\chi_{\mathrm{f}}^{3 \mathrm{FI}}$ spaces defined by Orlicz function, J. Fuzzy Math., 27 (2019), 707-721.

[12] V. A. Khan, K. M. A. S. Alshlool, S. A. A. Abdullah, Spaces of ideal convergent sequences of bounded linear operators, Numer. Funct. Anal. Optim., 39 (2018), 1278-1290.

[13] V. A. Khan, K. M. A. S. Alshlool, S. A. A. Abdullah, R. K. A. Rababah, A. Ahmad, Some new classes of paranorm ideal convergent double sequences of $\sigma$-bounded variation over n-normed spaces, Cogent Math. Stat., 5 (2018), 13 pages. 1

[14] V. A. Khan, K. Ebadullah, I-convergent difference sequence spaces defined by a sequence of moduli, J. Math. Comput. Sci., 2 (2012), 265-273. 1.5

[15] V. A. Khan, N. Khan, On Zweier I-convergent double sequence spaces, Filomat, 30 (2016), 3361-3369. 1

[16] V. A. Khan, A. A. H. Makharesh, K. M. A. S. Alshlool, S. A. A. Abdullah, H. Fatima, On fuzzy valued lacunary ideal convergent sequence spaces defined by a compact operator, J. Intel. Fuzzy Syst., 35 (2018), 4849-4855. 1

[17] V. A. Khan, R. K. A. Rababah, K. M. A. S. Alshlool, S. A. A. Abdullah, A. Ahmad, On Ideal convergence Fibonacci difference sequence spaces, Adv. Difference Equ., 2018 (2018), 14 pages. 1

[18] P. Kostyrko, M. Macaj, T. Šalát, Statistical convergence and I-convergence, Real Analysis Exchange, New York, (1999). $1,1.4$

[19] E. Malkowsky, Recent results in the theory of matrix transformations in sequence spaces, Mat. Vesnik, 49 (1997), 187-196. 1

[20] M. Mursaleen, F. Başar, B. Altay, On the Euler sequence spaces which include the spaces $\ell_{\mathrm{p}}$ and $\ell_{\infty}$ II, Nonlinear Anal., 65 (2006), 707-717.

[21] M. Mursaleen, A. K. Noman, On some new sequence spaces of non-absolute type related to the spaces $\ell_{p}$ and $\ell_{\infty} I$, Filomat, 25 (2011), 33-51. 1

[22] H. POLAT, Some new hilbert sequence spaces, Muş Alparslan Üniversitesi Fen Bilimleri Dergisi, 4 (2016), 367-372. 1

[23] T. Šalát, B. C. Tripathy, M. Ziman, On some properties of I-convergence, Tatra Mt. Math. Publ., 28 (2004), 279-286. 1, $1.3,1.6,1.7,1.8,1.9,1.10$

[24] T. Šalát, B. C. Tripathy, M. Ziman, On I-convergence field, Ital. J. Pure Appl. Math., 17 (2005), 45-54. 1, 1.11

[25] H. Steinhaus, Sur la convergence ordinaire et la convergence asymptotique, Colloq. Math., 2 (1951), 73-74. 1, 1.1

[26] N. Subramanian, A. Esi, On Triple sequence of Bernstein operator of weighted rough $\mathrm{I}_{\lambda}$-convergence, J. Class. Anal., 13 (2018), 45-62. 1

[27] N. Subramanian, A. Esi, V. A. Khan, The Rough Intuitionistic Fuzzy Zweier Lacunary Ideal Convergence of Triple Sequence spaces, J. Math. Stat., 14 (2018), 72-78.

[28] B. C. Tripathy, B. Hazarika, I-convergent sequence spaces associated with multiplier sequences, Math. Inequal. Appl., 11 (2008), 543-548. 1

[29] A. Wilansky, Summability through functional analysis, North-Holland Publishing Co., Amsterdam, (1984). 2 